\title{
Focusing on results after meniscus surgery
}

\author{
Philippe Beaufils · Roland Becker • Rene Verdonk • \\ Henrik Aagaard · Jon Karlsson
}

Published online: 20 December 2014

(C) European Society of Sports Traumatology, Knee Surgery, Arthroscopy (ESSKA) 2014

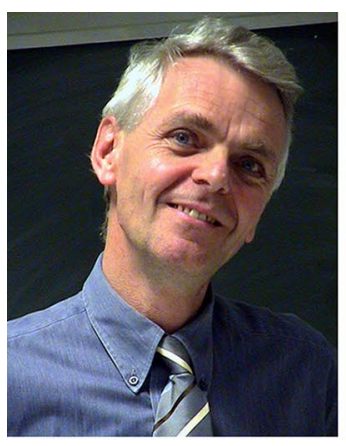

Phillippe Beaufils

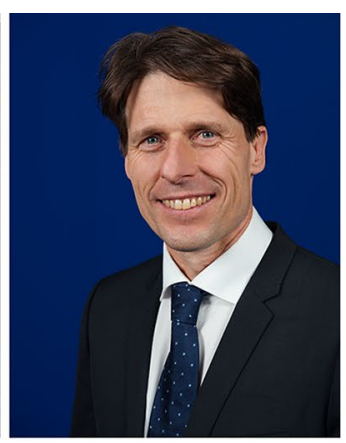

Roland Becker

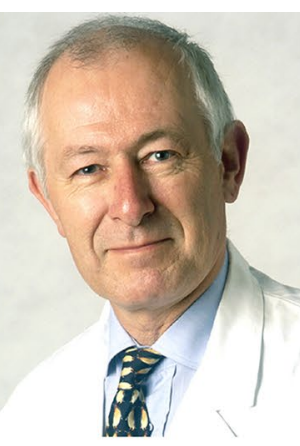

Rene Verdonk

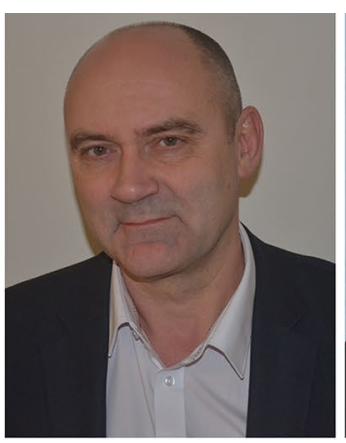

Henrik Aagaard

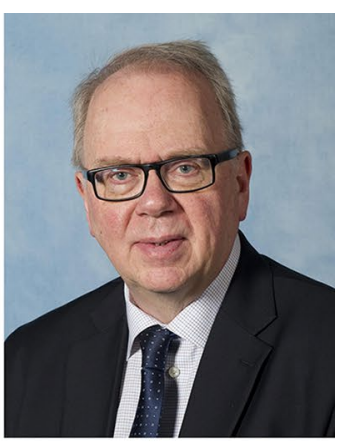

Jon Karlsson
Several randomized studies have recently been published and have sparked a highly controversial discussion among orthopaedic surgeons and others. The treatment of meniscal

\section{P. Beaufils $(\square)$}

Department of Orthopaedics and Traumatology, Centre

Hospitalier de Versailles, 78150 Le Chesnay, France

e-mail: pbeaufils@ch-versailles.fr

\section{R. Becker}

Department of Orthopaedics and Traumatology, Hospital

Brandenburg, Brandenburg, Germany

\section{R. Verdonk}

Department of Orthopaedics and Traumatology, University Ghent, Ghent, Belgium

\section{H. Aagaard}

Arthroscopic Centre, Amager-Hvidovre Hospital, Copenhagen, Denmark

J. Karlsson

Department of Orthopaedics, Sahlgrenska University Hospital, Göteborg, Sweden lesions has been moved into focus not only by many surgeons but also by the health authorities.

The current issue deals exclusively with the most recent knowledge of the pathology, diagnostics and management of meniscal lesions.

Until the 1990s, meniscal lesions were generally treated by total or partial meniscectomy, as the importance of the meniscus in terms of joint protection was still not well understood. This has changed primarily as a result of the development of arthroscopy and magnetic resonance imaging. Nowadays, an extensive knowledge of the meniscus and its importance to the healthy knee is available.

Along with technical developments, arthroscopic meniscectomy has become a great success, as a result of three main factors:

1. the speed of the arthroscopic procedure

2. low morbidity and

3. good short-term results.

The somewhat uncritical resection of the total meniscus was questioned, when anatomic and biomechanical studies 
clearly demonstrated that the meniscus is directly involved in the absorption of load, proprioception, stability and joint lubrication. This small piece of $\mathrm{C}$-shaped tissue appears to be very important when it comes to the prevention of early osteoarthritis. The era of meniscus preservation began and was based on three pillars:

- Repair of the torn meniscus whenever reasonable

- Conservative/non-surgical treatment of asymptomatic meniscal pathologies despite a meniscal tear according to MRI

- Partial meniscectomy and resection of as much as necessary and as little as possible.

Long-term results after arthroscopic meniscectomy are available. Almost all the studies conclude that meniscal repair is superior to meniscectomy in terms of function, return to sports and cartilage protection [15, 17-20, 25].

An improved long-term outcome and higher activity among patients were reported in a meta-analysis by Chen et al. [2] after meniscus repair in comparison with resection, basically summarizing the results of the current literature.

Longitudinal lesions of the meniscus in the vascularized region appear to be the ideal indication for meniscus repair. Keeping the above-mentioned knowledge in mind, less ideal lesions are also repaired nowadays, and studies of the current issue will present the outcome after meniscus repair in the avascular zone and of root tears, for example. These studies show the beneficial results of meniscal repair with regard to meniscus healing and function.

However, as the literature has proven the importance of the meniscus, how can we explain the small number of repairs in our daily practice? Meniscus repair was performed in $6.5 \%$ of all meniscus procedures in Germany in 2012 and in $5.6 \%$ in France in 2013 (ATIH, Agence Technique de l'Information sur l'Hospitalisation). In view of the $2.7 \%$ of meniscus repairs in France in 2008, the number has clearly evolved in the right direction, but it still remains low and under the 15-25\% of meniscus lesions, which appears to be appropriate for repair. These data show that meniscectomy will still be overused in 2015. How can we explain this gap?

The adoption of a surgical procedure by the majority of the surgical community is seldom only due to the quality of the scientific articles (evidence-based medicine). Other non-scientific criteria play a very important role when discussing the success of meniscus repair and meniscus resection. The following facts are, for example, well accepted.

1. Meniscal repair is technically more demanding.

2. Meniscal repair is more time-consuming.
3. Meniscal repair requires longer rehabilitation, which is less accepted by some of the patients due to professional or private commitments.

4. The economic constraints appear to have an impact on the method of surgical treatment, such as expensive implants, surgeons' fees that are similar to those received after meniscectomy for more difficult surgery and more physiotherapy.

Meniscal root tears have recently moved into focus. Should we repair these lesions? If so, which technique should we use for repair and what does the biomechanical evaluation tell us after the repair? The study by Röpke et al. [22], for instance, showed that, even after root repair, the deformation of the cartilage remains unnaturally high. Does the repair of these lesions really prevent or delay the development of early osteoarthritis?

Meniscal allografts or meniscal substitutes remain a difficult subject, but in this context, we are constantly improving our knowledge. Currently, meniscal reconstruction using an allograft or substitute should be considered as a salvage procedure after meniscectomy, especially when the lateral meniscus of a young patient is involved. A metaanalysis of meniscus allograft transplantation published by Smith et al. [26] showed the effectiveness of this treatment. However, further studies are needed in order to prove the chondroprotective effect of meniscus transplantation. One of the main concerns still appears to be the extrusion of the meniscus transplant, which may neutralize the protective function of the cartilage [4].

Let us now move to the middle-aged patient suffering from a symptomatic degenerative meniscus lesion.

The question of whether a meniscectomy in degenerative lesions should be routinely performed is still controversial and has generated an intensive worldwide debate [5, 13, 21]. The definition of the degenerative meniscal lesion and its relationship with the osteoarthritic process is now well defined [6]. The frequency of asymptomatic meniscal lesions in the elderly population should also be underlined. It should be stressed that the presence of a meniscal lesion on MRI does not necessarily cause symptoms.

What is the place of the meniscectomy in this context? The debate is open. Numerous publications reported functional improvements after meniscectomy but also the increased risk of osteoarthritis in the mid- and long-term perspective. Six randomized studies have recently questioned the legitimacy of arthroscopy in the degenerative joint or in isolated torn menisci [7, 9-12, 15, 24].

It started in 2002 when Moseley et al. [15] were the first to perform a randomized study comparing meniscectomy versus sham surgery in patients with knee osteoarthritis. The results were no better after meniscectomy compared 
with placebo surgery, but the patient selection and the documentation of intra-articular pathology have been criticized. Kirkley et al. [12] found similar results in a randomized controlled trial, comparing arthroscopic surgery with physiotherapy in osteoarthritic knees. Moreover, in that study, the inclusion criteria were questionable. However, does this mean that we should condemn arthroscopy in osteoarthritic knees? We do not think so.

Recently, Katz et al. [11], Herrlin et al. [9, 10] and Sihvonen et al. [24] questioned the efficacy of arthroscopic meniscectomy in degenerative meniscal lesions even in the absence of advanced signs of osteoarthritis. The first two studies compared partial meniscectomy with physiotherapy, while the third compared it with sham surgery.

All these publications need to be read with great care.

The main message in the paper by Katz et al. [11], for example, was that there is no difference between patients assigned to physiotherapy or arthroscopy. However, it should be noted that there was a crossover from the physiotherapy group to the arthroscopy group in $35 \%$ of the patients. The data have also shown that there was a failure rate of $49 \%$ in the physiotherapy group and $19 \%$ in the arthroscopy group. The result was found, in spite of the fact that more patients were suffering from Grade III OA in the arthroscopy group (28\%) than in the physiotherapy group $(25 \%)$. Did the authors really emphasize these findings appropriately? Arthroscopy is a successful therapy in patients suffering from meniscal tears and mild or moderate osteoarthritis, but this statement is not in agreement with the main statement given by the authors. The important finding in the study might be the fact that we should instead wait a while during a period of conservative treatment, before arthroscopy is considered. This would be a completely different message, as highlighted by the authors.

Sihvonen et al. [24] studied patients suffering from isolated degenerative meniscal tears but without signs of cartilage degeneration. How many of these patients have you seen in your daily practice? The final decision for inclusion was produced during arthroscopy, but the ICRS classification was not used to prove the correct inclusion criteria. Can we really exclude early degeneration of the knee when the assessment is based on the radiographic classification by Kellgren and Lawrence? The poor reliability and correlation between the radiographic grading and the actual histological findings are well known [16]. Moreover, the authors did not classify the meniscal tear in terms of size, shape and location, but all this information is important. The authors found a positive McMurray test in only $23 \%$ of the patients. However, the reliability of the test is reported to be approximately $85 \%$. What was the true problem in the patients with a negative McMurray sign? Do these data allow us to draw the conclusion that the outcome after arthroscopy in degenerative meniscal tears in patients without OA is similar to sham surgery? It is also important to remember that all patients received surgery, namely arthroscopic lavage or arthroscopy and meniscus resection. Arthroscopic lavage should not be called sham surgery. Why do the authors spend so much time discussing arthroscopy treatment versus conservative treatment? All patients received surgery in the study by Sihvonen et al.

At the same time, Gauffin et al. [7] performed a randomized study comparing arthroscopy with conservative treatment. The authors reported better results in the meniscectomized group in comparison with the physiotherapy group at the one-year follow-up, regardless of whether the design was "intention-to-treat analysis" or "as treated".

The important question is how the message of two level 1 scientific studies can be so different, taking account of the fact that the authors were focusing on the same clinical question?

Both Gauffin et al. [7] and Katz et al. [11] studied patients presenting with a symptomatic meniscus lesion in conjunction with a mild or moderate degree of osteoarthritis. Both of them included patients with joint space narrowing of less than $50 \%$ according to the Ahlbäck classification (Ahlbäck 0), as explicitly stated by both authors. The mean age of the patients of $54 \pm 6$ and $57 \pm 8$ years was similar in both studies.

This is an example showing that authors focusing on similar pathologies can still come to opposite findings and conclusions. We are wondering who is right? However, these studies show the enormous responsibility of both authors and editors when publishing scientific data. It is necessary to be somewhat more cautious when presenting results, which contradict what appears to be the current standard of care. The results of the studies by Katz et al. and Sihvonen et al. are important for the orthopaedic community and should be discussed seriously in order to identify the patients who require conservative treatment or meniscus surgery. However, it appears to be less helpful to generalize the findings.

There is no doubt that high-level clinical studies are very difficult to design and even more difficult to carry out, especially from an ethical point of view. The responsibility of the authors when publishing their data is very high.

What is the main message of these studies? The conclusion that the outcome of arthroscopy is similar to that of conservative treatment is incorrect and can easily be misunderstood. The studies have shown that there is no need for immediate arthroscopic surgery/meniscectomy and, in some of these patients, conservative treatment will be successful. Patients should undergo conservative treatment first, and surgery should be considered as a second line of treatment. However, this depends on the patient's symptoms.

We all are sure that there are a number of patients who may only require conservative treatment, but the way in which the data are presented in some of the above-mentioned studies is questionable. 
Two main lessons should be learned from this apparent contradiction.

1. A well-designed randomized study alone cannot be regarded as the sole and final truth. Numerous elements or biases (as we mention above) can contribute to the identical subject; thereby altering the results, e.g. conditions of cohort recruitment, definition of the selection criteria (what is a degenerative meniscal lesion?), role of the time between the onset of symptoms and the date of study inclusion (numerous symptomatic meniscal lesions become spontaneously asymptomatic in the first months). Data "beautification or embellishment" that enhances positive outcomes should not be forgotten either [23]. These biases have been repeatedly studied in the medical literature and consist of a selective choice of statistically significant results, inversion between the main and second objective, use of composite judgment criteria, false double-blind RCTs, false intention-totreat methodology, subgroups which are not included in the initial protocol and non-supported conclusions $[3,8$, 23]. Taken as a whole, in the Chess study [1] comprising 232 randomized studies in orthopaedic surgery, the authors conclude that a very few studies only meet all the criteria used for quality assessment of methodology in orthopaedics-related randomized controlled trials.... "Thus, many of these studies likely have biased estimates of treatment effects".

2. These studies, however solid they may appear, should be read and interpreted with a critical scientific mind. A single study can seldom be taken as the only fact or the final truth but should contribute to the scientific debate with the ultimate objective of improving our practice [14].

The necessity of a consensual process becomes clear, founded on the independence of the organizers and with the participation of all interested parties to produce the most exhaustive critical analysis of the literature possible. Work of this kind will permit a probable reduction in the number of arthroscopic meniscal resections in our countries in favour of abstention and meniscal repair and an improved nosological definition of the meniscectomy, rendering it pertinent and efficient.

Conflict of interest P.B. is Chief editor of Orthopaedics and Traumatology, Surgery and Research (Elsevier ed.).

\section{References}

1. Chess L, Gagnier J (2013) Risk of Bias of controlled trials published in orthopaedic journals. BMC Med Res Methodol 13:76-85

2. Chen W, Zhao J, Wen Y, Xie B, Zhou X, Guo L, Yang L, Wang J, Dai Y, Zhou D (2014) Accuracy of 3-T MRI using susceptibility-weighted imaging to detect meniscal tears of the knee. Knee Surg Sports Traumatol Arthrosc. doi:10.1007/ s00167-014-3035-0

3. Clavien PA, Puhan MA (2014) Biased reporting in surgery. Wiley Online Library. doi:10.1002/bjs.9499, www.bjs.co.uk

4. De Coninck T, Huysse W, Verdonk R, Verstraete K, Verdonk P (2013) Open versus arthroscopic meniscus allograft transplantation: magnetic resonance imaging study of meniscal radial displacement. Arthroscopy 29(3):514-521

5. El Attrache N, Lattermann C, Hannon M, Cole B (2014) New England Journal of Medicine Article evaluating the usefulness of meniscectomy is flawed. Arthroscopy 30(5):542-543

6. Englund M, Roemer FW, Hayashi D, Crema MD, Guermazi A (2012) Meniscus pathology, osteoarthritis and the treatment controversy. Nat Rev Rheumatol 8(7):412-419

7. Gauffin H, Tagesson S, Meunier A, Magnusson H, Kvist (2014) Knee arthroscopic surgery is beneficial to middle-aged patients with meniscal symptoms: a prospective, randomised, singleblinded study. Osteoarthr Cartil. doi:10.1016/j.joca.2014.07.017

8. Hannink G, Gooszen H, Rovers M (2013) Comparison of registered and published primary outcomes in randomized clinical trials of surgical interventions. Ann Surg 257(5):818-823

9. Herrlin S, Hållander M, Wange P, Weidenhielm L, Werner S (2007) Arthroscopic or conservative treatment of degenerative medial meniscal tears: a prospective randomised trial. Knee Surg Sports Traumatol Arthrosc 15(4):393-401

10. Herrlin SV, Wange PO, Lapidus G, Hållander M, Werner S, Weidenhielm L (2013) Is arthroscopic surgery beneficial in treating non-traumatic, degenerative medial meniscal tears? A five year follow-up. Knee Surg Sports Traumatol Arthrosc 21(2):358-364

11. Katz JN, Brophy RH, Chaisson CE et al (2013) Surgery versus physical therapy for a meniscal tear and osteoarthritis. N Engl J Med 368(18):1675-1684

12. Kirkley A, Birmingham TB, Litchfield RB et al (2008) A randomized trial of arthroscopic surgery for osteoarthritis of the knee. N Engl J Med 359(11):1097-1107

13. Lubowitz JH, Provencher MT, Rossi MJ (2014) Could the New England Journal of Medicine be biased against arthroscopic knee surgery? Arthroscopy 30(6):654-655

14. McIntyre LF, Beach WR, Higgins LD et al (2013) Evidence based medicine, appropriate use criteria, and sports medicine: how best to develop meaningful treatment guidelines. Arthroscopy 29(7):1224-1229

15. Moseley JB, O’Malley K, Petersen NJ (2002) A controlled trial of arthroscopic surgery for osteoarthritis of the knee. $\mathrm{N}$ Engl $\mathrm{J}$ Med 347(2):81-88

16. Nebelung S, Marx U, Brill N et al (2014) Morphometric grading of osteoarthritis by optical coherence tomography: an ex vivo study. J Orthop Res 32(10):1381-1388

17. Nepple JJ, Dunn WR, Wright RW (2012) Meniscal repair outcomes at greater than five years: a systematic literature review and meta-analysis. J Bone Joint Surg Am 94(24):2222-2227

18. Paxton ES, Stock MV, Brophy RH (2011) Meniscal repair versus partial meniscectomy: a systematic review comparing reoperation rates and clinical outcomes. Arthroscopy 27:1275-1288

19. Pujol N, Beaufils P (2009) Healing results of meniscal tears left in situ during anterior cruciate ligament reconstruction: a review of clinical studies. Knee Surg Sports Traumatol Arthrosc 17:396-400

20. Pujol N, Tardy N, Boisrenoult P, Beaufils P (2013) Long-term outcomes of all-inside meniscal repair. Knee Surg Sports Traumatol Arthrosc. doi:10.1007/s00167-013-2553-5

21. Rossi MJ, D'Agostino RB Jr, Provencher MT, Lubowitz JH (2014) Could the New England Journal of Medicine be biased against arthroscopic knee surgery? Arthroscopy 30(5):536-537

22. Röpke EF, Kopf S, Drange S, Becker R, Lohmann CH, Stärke Ch (2013) Biomechanical evaluation of meniscal root repair: 
a porcine study. Knee Surg Sports Traumatol Arthrosc. doi:10.1007/s00167-013-2589-6

23. Seror R, Ravaud P (2012) Beautification of data: minimal fraud, incompetence or mixture of both. Presse Med 41(9 Pt 1): 835-840

24. Sihvonen R, Paavola M, Malmivaara A et al (2013) Arthroscopic partial meniscectomy versus sham surgery for a degenerative meniscal tear. N Engl J Med 369(26):2515-2524
25. Stein T, Mehling AP, Welsch F et al (2010) Long-term outcome after arthroscopic meniscal repair versus arthroscopic partial meniscectomy for traumatic meniscal tears. Am J Sports Med 38:1542-1548

26. Smith NA, MacKay N, Costa M, Spalding T (2014) Meniscal allograft transplantation in a symptomatic meniscal deficient knee: a systematic review. Knee Surg Sports Traumatol Arthrosc. doi:10.1007/s00167-014-3310-0 